\title{
Catalytic Addition of Nitroalkanes to Unactivated Alkenes via Directed Carbopalladation
}

\author{
Amit Kumar Simlandy, Warabhorn Rodphon, Hui-Qi Ni, John A. Gurak, Jr., Keary M. Engle*
}

[a] Dr. Amit Kumar Simlandy, Warabhorn Rodphon, Hui-Qi Ni, John A. Gurak, Jr., and Prof. K. M. Engle

Department of Chemistry

The Scripps Research Institute

10550 N. Torrey Pines Road, La Jolla, CA 92037, USA

E-mail: keary@scripps.edu

Supporting information for this article is given via a link at the end of the document

\begin{abstract}
We report a redox-neutral catalytic coupling of nitroalkanes and unactivated alkenes that proceeds by a directed carbopalladation mechanism. The reaction is uniquely enabled by the combination of $\mathrm{Pdl}_{2}$ as the precatalyst and HFIP solvent. Structurally complex nitroalkane products, including nitro-containing carbo- and heterocycles, are prepared under operationally convenient conditions without the need for toxic or corrosive reagents. Deuterium labeling experiments and isolation of a catalytically relevant intermediate shed light on the reaction mechanism. By taking advantage of different catalytic activation modes, we demonstrate orthogonal methods for site-selective functionalization of a polyfunctional nitroalkyl ketone.
\end{abstract}

The nitro group is a versatile synthetic handle that can easily be transformed into other useful functional groups. ${ }^{[1]}$ Nitroalkanes are a readily available and shelf-stable family of nitrated synthetic building blocks that commonly serve as pronucleophiles; upon deprotonation at the $\mathrm{a}-\mathrm{C}-\mathrm{H}$ bond $\left(\mathrm{MeNO}_{2} \mathrm{pK}\right.$ a $\left.(\mathrm{DMSO})=17.2\right)$, the resulting nitronate anion is able to engage various carbon-based electrophiles to enable $\mathrm{C}-\mathrm{C}$ bond formation. Compatible electrophiles include, carbonyl compounds (Henry reaction), ${ }^{[2]}$ enones and other conjugated alkenes (Michael addition), ${ }^{[3]}$ and organometallic species generated from oxidative addition of a low-valent metal catalyst to allyl electrophiles ${ }^{[4]}$ or aryl/alkyl halides (Figure 1A). ${ }^{[5]}$ Expansion of this toolkit to unactivated alkenes through a catalytic $\pi$-Lewis acid activation mechanism would broaden the scope of nitro-containing product structures that could be accessed, yet such a transformation remains unknown to date.

In recent years, substrate-directed Heck- and Wackertype alkene addition reactions have gained prominence as enabling tools in synthesis. $\left.{ }^{[6,} 8,10\right]$ We have previously employed 1,3-dicarbonyl compounds and other $\mathrm{C}-\mathrm{H}$ pronucleophiles in a $\mathrm{pK}$ a range of 10-18 in directed carboWacker-type additions to alkenes under $\mathrm{Pd}(\mathrm{II})$ catalysis. ${ }^{[6 \mathrm{~b}]}$ Based on this precedent we questioned whether it would be possible to enlist nitroalkanes in this type of process (Figure 1B). ${ }^{[7]}$ Successful realization of such a catalytic coupling would be practically significant as it would facilitate expedient synthesis of highly substituted nitroalkane products without the need for harsh reagents (e.g., nitric acid). Conceptually, it would demonstrate the feasibility of using nitrated nucleophiles in the modular approach to alkene difunctionalization that our lab and others have developed during the past five years. ${ }^{[8]}$
A. $\alpha$-Functionalization of nitroalkanes for $C-C$ bond formation

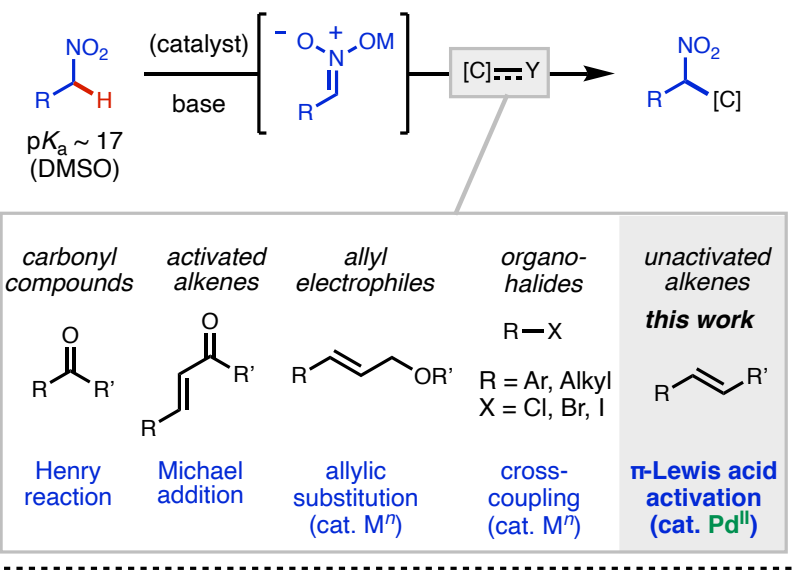

B. This Work: Carbo-Wacker nitroalkane addition

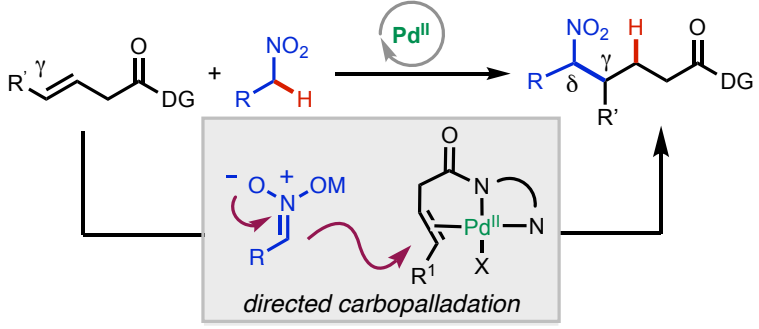

Figure 1. Background and envisioned transformation

To reduce this idea to practice, we initially focused on the model reaction shown in Table 1, in which a 3-butenoic acid substrate containing an 8-aminoquinoline amide (AQ) directing auxiliary (1a) is coupled with excess nitroethane (2a) (10 equiv). Pilot experiments revealed that published conditions for 1,3dicarbonyl nucleophiles were unsuccessful in this case (entry 2). ${ }^{[6]}$ After extensive optimization, we identified optimal conditions consisting of $\mathrm{Pdl}_{2}$ as catalyst, $\mathrm{Na}_{2} \mathrm{CO}_{3}$ as base, and HFIP as solvent at $80{ }^{\circ} \mathrm{C}$ (entry 1 ). The presence of iodide as the counteranion was found to be critical, as other commonly used $\mathrm{Pd}(\mathrm{II})$ sources like $\mathrm{PdCl}_{2}$ and $\mathrm{Pd}(\mathrm{OAc})_{2}$ were ineffective (entries 3-5). Other inorganic bases like $\mathrm{K}_{2} \mathrm{CO}_{3}$ were found to be inferior (entry 6 ). While the reaction provided synthetically useful yields with as low as 2.0 equiv $\mathrm{EtNO}_{2}$, the yield steadily decreased with lower loadings of $\mathrm{EtNO}_{2}$ (entries 7-8). Fluorinated alcohol solvents were found to be uniquely effective in promoting this coupling (entries 9-12), while aprotic 
solvents with different dielectric constants (entry 9) and nonfluorinated polar protic solvents (e.g., ${ }^{t} \mathrm{BuOH}$ and ${ }^{t} \mathrm{AmOH}$ ) (entry 10) failed to facilitate the reaction. Compared to HFIP, TFE and $\left(\mathrm{CF}_{3}\right)_{3} \mathrm{COH}$ were competent though led to lower yield (entries 11-12).

Table 1. Reaction optimization

\begin{tabular}{|c|c|c|c|}
\hline$\underbrace{\mathrm{O}}_{1 \mathrm{1}}$ & $\mathrm{Me}_{\text {(10 equiv) }}^{\mathrm{NO}_{\mathrm{H}}^{\mathrm{NO}_{2}}}$ & $\begin{array}{c}\mathrm{Pdl}_{2}(10 \mathrm{~mol} \%) \\
\mathrm{Na}_{2} \mathrm{CO}_{3}(1.0 \text { equiv })\end{array}$ & $\underbrace{H}_{3 a a}$ \\
\hline entry & \multicolumn{2}{|c|}{ deviation from standard conditions } & yield $(\%)^{[a]}$ \\
\hline 1 & & None & $83^{[b]}$ \\
\hline 2 & $\mathrm{AcOH}, \mathrm{Pc}$ & $\mathrm{OAC})_{2}, \mathrm{MeCN}, 120^{\circ} \mathrm{C}$ & $<10^{\text {[ref. } 6 \mathrm{~b}]}$ \\
\hline 3 & $\mathrm{PdC}$ & instead of $\mathrm{Pdl}_{2}$ & 11 \\
\hline 4 & $\mathrm{Pd}(\mathrm{O}$ & c) $)_{2}$ instead of $\mathrm{Pdl}_{2}$ & 13 \\
\hline 5 & & $(\mathrm{OAc})_{2}, \mathrm{TBAI}$ & 60 \\
\hline 6 & $\mathrm{~K}_{2} \mathrm{CO}_{3}$ & instead of $\mathrm{Na}_{2} \mathrm{CO}_{3}$ & 36 \\
\hline 7 & 5.0 & quiv. of $\mathrm{EtNO}_{2}$ & 57 \\
\hline 8 & 2.0 & quiv. of $\mathrm{EtNO}_{2}$ & 37 \\
\hline 9 & Toluene or & MeCN instead of HFIP & $<10$ \\
\hline 10 & ${ }^{t} \mathrm{BuOH}$ or & $\mathrm{ImOH}$ instead of HFIP & $<10$ \\
\hline 11 & $\left(\mathrm{CF}_{3}\right)_{3} \mathrm{C}$ & $\mathrm{OH}$ instead of HFIP & 50 \\
\hline 12 & TFE & instead of HFIP & 63 \\
\hline
\end{tabular}

[a] ${ }^{1} \mathrm{H}$ NMR yield of the crude reaction mixture was determined using mesitylene as an internal standard. [b] Isolated yield, reaction time $6 \mathrm{~h}$.

Having identified optimal reaction conditions, the scope of the method was next evaluated (Scheme 1). A diverse collection of nitroalkane coupling partners participated in the reaction. It is important to mention that for valuable nitroalkanes, use of either 5.0 equiv or 2.0 equiv was sufficient to generate the nitro-containing products in good to excellent yields. Beyond the bulk chemicals nitroethane (3aa) and nitromethane (3ab), more structurally complex nitroalkane coupling partners were effective (3ac-3aj), including those containing potentially reactive or inhibitory functional groups, such as electron-rich heterocycles (3ad), ketones (3ae), esters (3af), amides (3ag), alkenes (3ah), protected amines (3ai), and alkynes (3aj). Regarding the alkene scope, substituents were well tolerated at the position $\alpha$ - with respect to the amide directing group (3ba-3da), albeit with variable d.r. An internal alkene was competent, though the yield was low (3eb). A number of limitations were also identified, as a,a-disubstituted nitroalkanes and substrates with longer spacers between the alkene and directing group were unreactive.

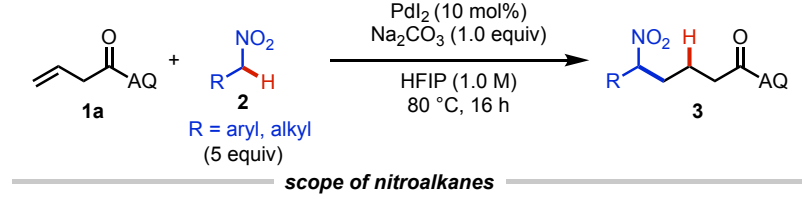

$$
\text { 3aa, }(83 \%)^{[b]}
$$
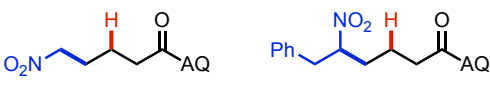

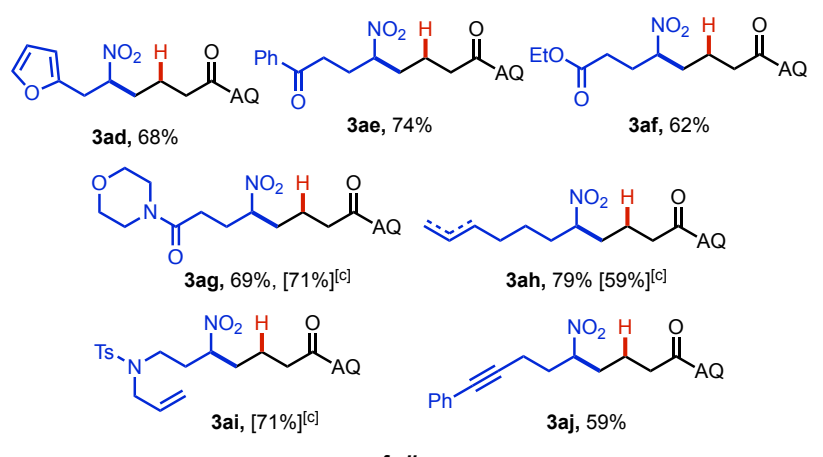

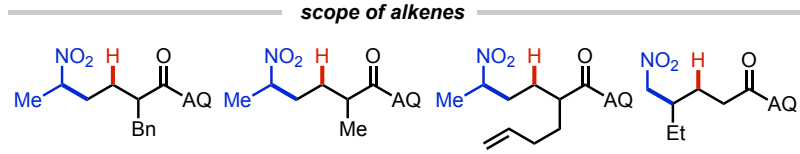

3ba, 39\%, 80:20 dr $\quad 3$ ca, 60\%, 88:12 dr $\quad$ 3da, 73\%, 50:50 dr $\quad 3$ eb, $20 \%$

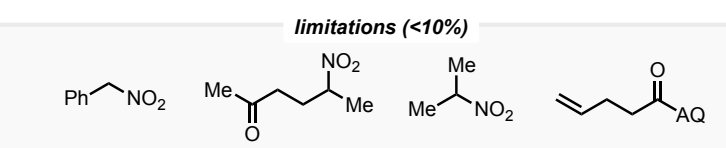

[a] Percentages represent isolated yields unless otherwise specified. [b] The values in arentheses correspond to expeiments with 10 equiv nitroalkane, reaction time 6 h. (2). [c] The values in brackets correspond to experiments with 2 equiv nitroalkane (2).

Scheme 1. Substrate and coupling partner scope. ${ }^{[a]}$

By using cross-metathesis ${ }^{[9]}$ it was possible to quickly access substrates in which the nitroalkane is intramolecularly tethered to the alkene. Under the optimal conditions from Table 1 , redox-neutral carbocyclization ${ }^{[6,10]}$ took place to furnish nitro-substituted carbo- and heterocycles in moderate yield and with good to excellent diastereoselectivity (Scheme 2). ${ }^{[11]}$ An X-ray crystal structure of $\mathbf{5 a}$ confirmed the trans relationship between the nitro group and the alkyl chain containing $A Q$ in the major product.

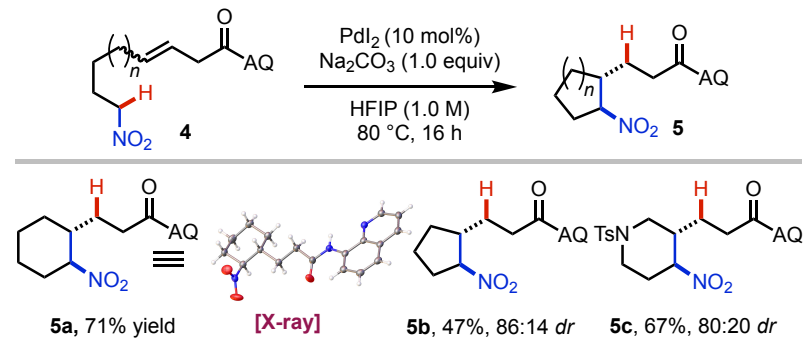

Scheme 2. Scope of intramolecular cyclization.

We investigated keto-nitroalkane $\mathbf{2 k}$ as a model bispronucleophile with different potential reaction sites that we thought could be addressed in a chemodivergent manner 
(Scheme 3A). Indeed, when $\mathbf{2} \mathbf{k}$ was subjected to the optimized reaction conditions, 3ak was obtained in $78 \%$ yield, with sites adjacent to the ketone unperturbed. Alternatively, a dual $\mathrm{Pd}(\mathrm{II}) /$ organocatalytic activation strategy ${ }^{[\mathrm{[i}, 6 \mathrm{j}, \text { 8j] }}$ was applied to unlock reactivity $\alpha$ to the ketone through enamine catalysis, and the product 3ak' was obtained in $69 \%$ yield as a $1: 1$ regioisomeric mixture with both a-positions proving to be reactive. Efforts to improve the regioisomeric ratio through optimization of the reaction conditions were unsuccessful (see ESI). To demonstrate how the nitroalkane pronucleophiles can serve as synthetic equivalents for other useful synthons, we performed a Nef reaction ${ }^{[12]}$ with $3 a a$ and were pleased to see the formation of $\mathbf{6}$, the product of formal hydroacylation of $\mathbf{1 a}$, in $87 \%$ yield (Scheme $3 \mathrm{~B}$ ). Alternatively, the nitro group could also be easily reduced under $\mathrm{Zn} / \mathrm{AcOH}$ conditions, and in the presence of a pendant ester, in situ cyclization took place to furnish compound 7 , representing a formal a-aminoalkyl anion addition (Scheme $3 \mathrm{C}$ ). The $\mathrm{AQ}$ protecting group in 3ac was easily removed by $\mathrm{Ni}(\mathrm{TMHD})_{2}$ in presence of methanol to obtain ester 8 (Scheme 3D). ${ }^{[13]}$

A
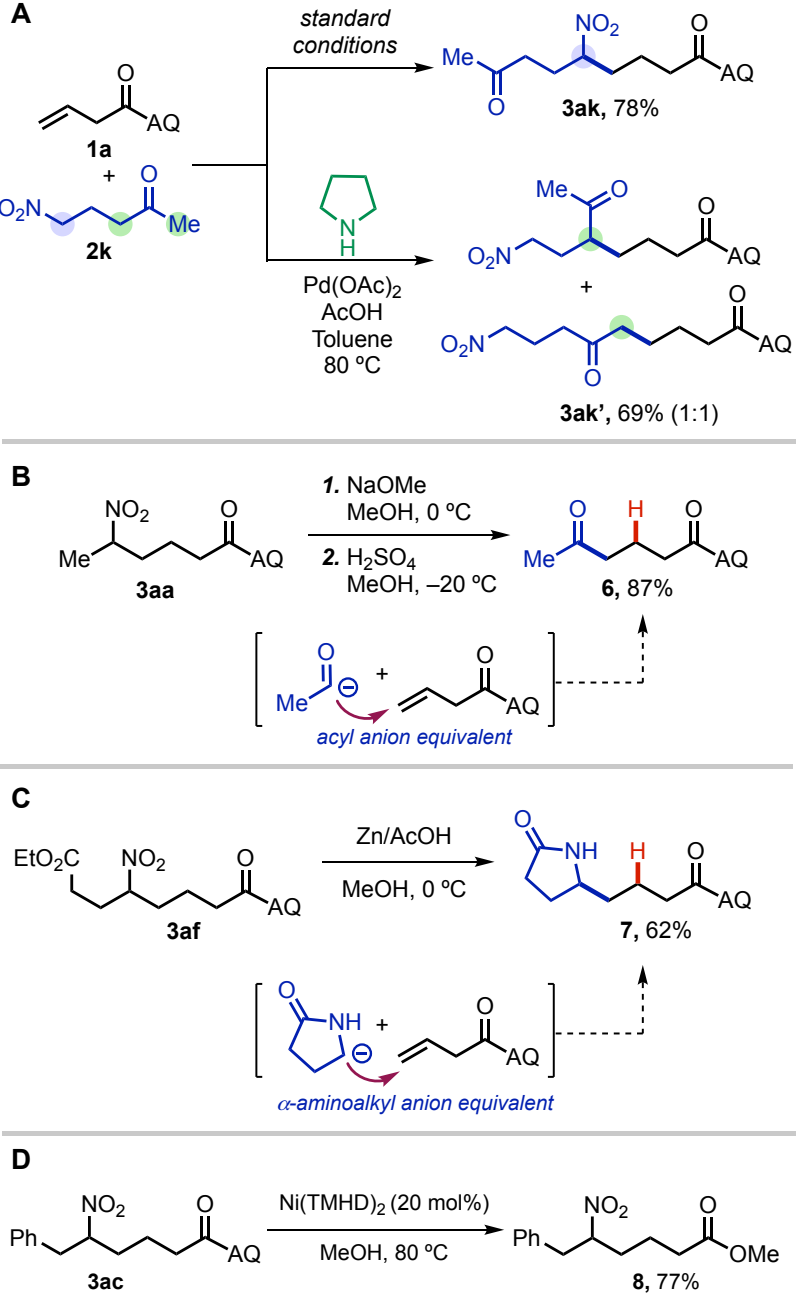

Scheme 3. $[A]$ Chemodivergent addition of nitroalkane. [B] Nef reaction. [C] Reduction. [D] Removal of $A Q$ protecting group. $T M H D=2,2,6,6-$ tetramethyl-3,5-heptanedionate.
To shed light on the mechanistic details of this transformation, particularly the importance of HFIP as solvent and $\mathrm{Pdl}_{2}$ as catalyst (Scheme 4), several experiments were performed. By treating 1a with stoichiometric $\mathrm{Pd}(\mathrm{OAc})_{2}$ and $\mathrm{Nal}$ in DCE, we were able to prepare substrate-bound $\mathrm{Pd}(\mathrm{II})$ complex Pd-1 and determine its X-ray crystal structure. ${ }^{[6 b]}$ This complex was found to be catalytically competent in the reaction, consistent with the notion that it may be an intermediate on the catalytic cycle.
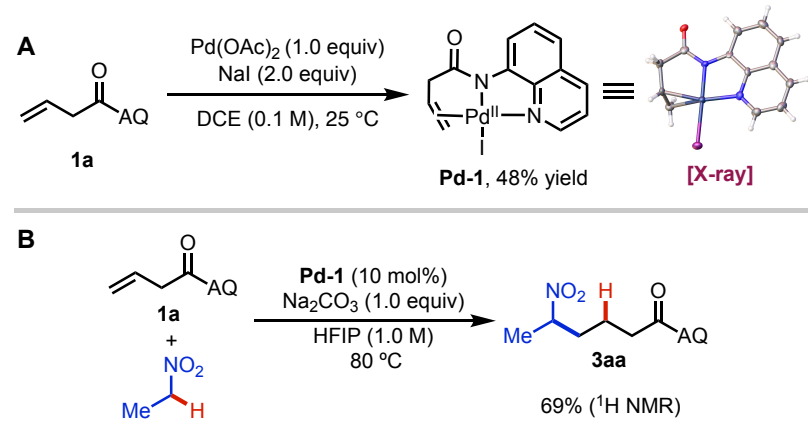

2a (10 equiv)

Scheme 4. [A] Synthesis of substrate-bound palladium complex Pd-1. [B] Assessment of the catalytic competence of Pd-1.

We then focused on determining the main source of the $\mathrm{H}$-atom in the product with a series of deuterium labeling experiments. Reactions were halted at low conversion to avoid complications from secondary H/D exchange processes (see $\mathrm{SI})$. Whereas use of deuterated solvent, $\left(\mathrm{CF}_{3}\right)_{2} \mathrm{CHOD}$, led primarily to $D$-incorporation at the $\delta$ position (directly adjacent to the nitro group), when the reaction was performed with $\mathrm{CD}_{3} \mathrm{NO}_{2}\left(\mathbf{2} \mathbf{b}-\boldsymbol{d}_{3}\right)$ as nucleophile, $21 \% \mathrm{D}$-incorporation at the $\beta$ position was observed. In both cases $H / D$ exchange at the alkenyl positions of the substrate ${ }^{[6]}$ was comparatively slow. This data indicates that the acidic protons/deuterons of the solvent and nucleophile do not freely exchange under the reaction conditions and also that the nitroalkane serves the dual role as nucleophile and proton/deuteron source in this catalytic alkene hydrofunctionalization. Given that HFIP is not the primary proton source in the reaction, a potential explanation for its role in promoting the reaction is stabilizing the charged sodium nitronate anion. ${ }^{[14]}$ Meanwhile, iodide may play a role as a Lewis basic site for sodium coordination to template nucleophilic attack. ${ }^{[15]}$
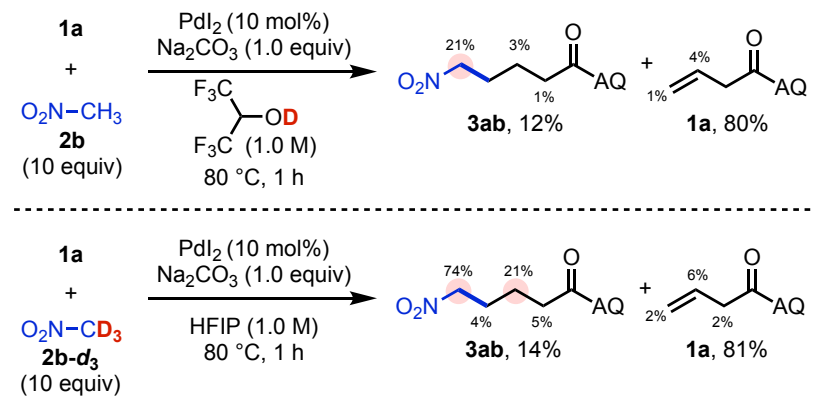

Scheme 5. Deuterium labelling study 
Based on previous reports ${ }^{[6,8]}$ and the mechanistic experiments above, a plausible catalytic cycle is drawn in Figure 2. First, Pd-1 complex is formed via the ligand exchange between $\mathrm{Pdl}_{2}$ and substrate (1a). Next, nucleopalladation delivers an alkyl palladacycle. ${ }^{[6]}$ This conformationally constrained is resistant to $\beta-\mathrm{H}$ elimination and is instead intercepted by an acidic proton originating from the nitroalkane to furnish the product (3ab). During this cycle acidic protons will be exchanged with deuterium in the presence of base.

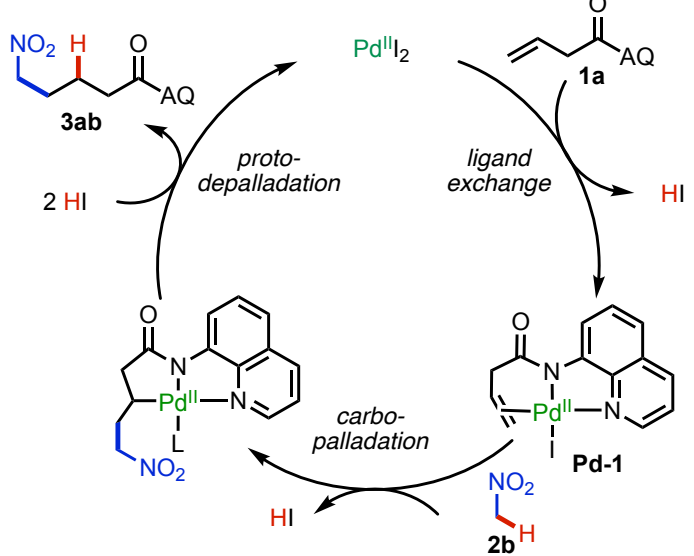

Figure 2. Plausible catalytic cycle with a representative alkene and nitroalkane.

In conclusion, we have developed a redox-neutral addition of nitroalkanes to unactivated alkenes via a directed nucleopalladation strategy, granting convenient access to various nitro-containing products in regioselective fashion. Whereas previously published protocols are incompatible with nitroalkane pronucleophiles, the discovery of specially tailored conditions comprised of $\mathrm{Pdl}_{2}$ as catalyst in HFIP as solvent allows for selective engagement of sites adjacent to nitro groups. In this way, chemodivergent addition of ketonitroalkane was demonstrated under different activation modes.

\section{Acknowledgements}

Financial support for this work was provided by the Office of Naval Research (N00014-20-1-2606). We further acknowledge the National Research Council of Thailand (NRCT) (NRCT5-RGJ63023-177, predoctoral fellowship to W.R.) and the U.S. National Science Foundation (NSF/DGE1346837, predoctoral fellowship to J.A.G.). We thank Dr. Milan Gembicky and Prof. Arnold L. Rheingold for X-ray crystallographic analysis.

Keywords: palladium $\cdot$ directing group $\cdot$ nitroalkane $\cdot$ alkene functionalization

[1] a) G. Rosini, R. Ballini, Synthesis 1988, 833-847. b) Ono, N. The Nitro Group in Organic Synthesis; Wiley-VCH: Weinheim, New York, 2001.

[2] a) F. A. Luzzio, Tetrahedron 2001, 57, 915-945. b) N. Singh, J. Pandey, Mini-Rev. Org. Chem. 2020, 17, 297-308.
[3] R. Ballini, G. Bosica, D. Fiorini, A. Palmieri, M. Petrini, Chem. Rev. 2005, 105, 933-972.

[4] a) P. Aleksandrowicz, H. Piotrowska, W. Sas, Tetrahedron 1982, 38 1321-1327. b) J. P. Genet, S. Grisoni, Tetrahedron Lett. 1988, 29 4543-4546. c) H. Rieck, G. Helmchen, Angew. Chem. 1995, 107 2881-2883; Angew. Chem. Int. Ed. 1996, 34, 2687-2689. d) B. M. Trost, J.-P Surivet, J. Am. Chem Soc. 2000, 122, 6291-6292 e) B. M. Trost, J.-P. Surivet, Angew. Chem. 2000, 112, 3252-3254; Angew. Chem. Int. Ed. 2000, 39, 3122-3124. f) T. Nemoto, L. Jin, H. Nakamura, Y. Hamada, Tetrahedron Lett. 2006, 47, 6577-6581. g) Y. Uozumi, T. Suzuka, J. Org. Chem. 2006, 71, 8644-8646. h) K. Maki, M. Kanai, M. Shibasaki, Tetrahedron 2007, 63, 4250-4257. i) A. J. Grenning, J. A Tunge, Angew. Chem. 2011, 123, 1726-1729; Angew. Chem. Int. Ed. 2011, 50, 1688-1691. j) X.-F. Yang, C.-H. Ding, X.-H. Li, J.-Q. Huang X.-L. Hou, L.-X. Dai, P.-J. Wang, J. Org. Chem. 2012, 77, 8980-8985. k) À. Cristòfol, E. C. Escudero-Adán, A. W. Kleij, J. Org. Chem. 2018, 83, 9978-9990. I) C.-Y. Chang, Y.-K. Wu, J. Org. Chem. 2018, 83, 6217-6224. m) C. Zhao, B. H. Shah, I. Khan, Y. Kan, Y. J. Zhang, Org. Lett. 2019, 21, 9045-9049. For iridium-catalyzed asymmetric allylic alkylation of nitro compounds, see: n) A. Dahnz, G. Helmchen, Synlett 2006, 697-700.

[5] For aliphatic electrophiles, see: a) A. A. S. Gietter, P. G. Gildner, A P. Cinderella, D. A. Watson, Org. Lett. 2014, 16, 3166-3169. b) K. W. Shimkin, P. G. Gildner, D. A. Watson, Org. Lett. 2016, 18, 988-991. c) A. A. S. Gietter-Burch, V. Devannah, D. A. Watson, Org. Lett. 2017, 19, 2957-2960. d) S. Rezazadeh, V. Devannah, D. A. Watson, J. Am Chem. Soc. 2017, 139, 8110-8113. e) V. Devannah, R. Sharma, D. A Watson, J. Am. Chem. Soc. 2019, 141, 8436-8440. f) R. S. Kim, L. V. Dinh-Nguyen, K. W. Shimkin, D. A. Watson, Org. Lett. 2020, 22, 81068110. For aryl electrophiles, see: g) R. S. Fornicola, E. Oblinger, J. Montgomery, J. Org. Chem. 1998, 63, 3528-3529. h) H. Muratake, H. Nakai, Tetrahedron Lett. 1999, 40, 2355-2358. i) J. M. Fox, X. Huang, A. Chieffi, S. L. Buchwald, J. Am. Chem. Soc. 2000, 122, 1360-1370. j) E. M. Vogl, S. L. Buchwald, J. Org. Chem. 2002, 67, 106-111. k) A E. Metz, S. Berritt, S. D. Dreher, M. C. Kozlowski, Org. Lett. 2012, 14 760-763. I) R. R. Walvoord, S. Berritt, M. C. Kozlowski, Org. Lett. 2012, 14, 4086-4089. m) R. R. Walvoord, M. C. Kozlowski, J. Org. Chem. 2013, 78, 8859-8864. n) K. F. VanGelder, M. C. Kozlowski, Org. Lett. 2015, 17, 5748-5751. o) J. Brals, J. D. Smith, F. Ibrahim, F. Gallou, S. Handa, ACS Catal. 2017, 7, 7245-7250. p) P.-F. Zheng, Y. An, Z.-Y. Jiao, Z.-B. Shi, F.-M. Zhang, Current Org. Chem. 2019, 23, 1560-1580.

[6] For hydrofunctionalizations, see: a) J. A. Gurak, Jr., K. S. Yang, Z. Liu, K. M. Engle, J. Am. Chem. Soc. 2016, 138, 5805-5808. b) K. S. Yang, J. A. Gurak, Jr., Z. Liu, K. M. Engle, J. Am. Chem. Soc. 2016 138, 14705-14712. c) J. A. Gurak, Jr., V. T. Tran, M. M. Sroda, K. M. Engle, Tetrahedron 2017, 73, 3636-3642. d) H. Wang, Z. Bai, T. Jiao, Z. Deng, H. Tong, G. He, Q. Peng, G. Chen, J. Am. Chem. Soc. 2018 140, 3542-3546. e) C. Wang, G. Xiao, T. Guo, Y. Ding, X. Wu, T.-P Loh, J. Am. Chem. Soc. 2018, 140, 9332-9336. f) R. Matsuura, T. C. Jankins, D. E. Hill, K. S. Yang, G. M. Gallego, S. Yang, M. He, F. Wang, R. P. Marsters, I. McAlpine, K. M. Engle, Chem. Sci. 2018, 9, 83638368. g) C. Wang, G. Xiao, T. Guo, Y. Ding, X. Wu, T.-P. Loh, J. Am. Chem. Soc. 2018, 140, 9332-9336. h) S. K. Nimmagadda, M. Liu, M. K. Karunananda, D.-W. Gao, O. Apolinar, J. S. Chen, P. Liu, K. M. Engle, Angew. Chem. 2019, 131, 3963-3967; Angew. Chem. Int. Ed. 2019, 58, 3923-3927. i) H.-C. Shen, L. Zhang, S.-S. Chen, J. Feng, B.W. Zhang, Y. Zhang, X. Zhang, Y.-D. Wu, L.-Z. Gong, ACS Catal. 2019 9, 791-797. j) C. Wei, X. Ye, Q. Xing, Y. Hu, Y. Xie, X. Shi, Org. Biomol. Chem. 2019, 17, 6607-6611. k) Z. Bai, Z. Bai, F. Song, H. Wang, G. Chen, G. He, ACS Catal. 2020, 10, 933-940. I) X. Wang, Z.-Q. Li, B. K. Mai, J. A. Gurak, Jr., J. E. Xu, V. T. Tran, H.-Q. Ni, Z. Liu, Z. Liu, K. S. Yang, R. Xiang, P. Liu, K. M. Engle, Chem. Sci. 2020, 11, 11307-11314. m) T. Guo, Y. Ding, L. Zhou. H. Xu, T.-P. Loh, X. Wu, ACS Catal. 2020, 10, 7262-7268. n) M.-Z. Lu, H. Luo, Z. Hu, C. Shao, Y. Kan, T.-P. Loh, Org. Lett. 2020, 22, 9022-9028. o) K. Zheng, G. Xiao, T. Guo, Y. Ding, C. Wang, T.-P. Loh, X. Wu, Org. Lett. 2020, 22, 694-699. p) C.-F. Zhu, C.-H. Gao, W.-J. Hao, Y.-L. Zhu, S.-J. Tu, D.-C. Wang, B. Jiang, Org Chem. Front. 2021, 8, 127-132. q) R. K. Shukla, A. K. Chaturvedi, S Pal, C. M. R. Volla, Org. Lett. 2021, 23, 1440-1444. r) R. K. Shukla, A. K. Chaturvedi, C. M. R. Volla, ACS Catal. 2021, 11, 7750-7761. s) L. Xia, Y. Wu, C. Lin, F. Gao, L. Shen, Asian J. Org. Chem. 2021 e202100742. 
[7] One example of a double activated $\alpha-\mathrm{NO}_{2}$ carbonyl pronucleophile was reported in Ref. $6 b$.

[8] For difunctionalizations, see: a) Z. Liu, T. Zeng, K. S. Yang, K. M. Engle, J. Am. Chem. Soc. 2016, 138, 15122-15125. b) Z. Liu, Y. Wang, Z. Wang, T. Zeng, P. Liu, K. M. Engle, J. Am. Chem. Soc. 2017, 139, 11261-11270. c) Z. Liu, H.-Q. Ni, T. Zeng, K. M. Engle, J. Am. Chem. Soc. 2018, 140, 3223-3227. d) T. Zeng, Z. Liu, M. A. Schmidt, M. D. Eastgate, K. M. Engle, Org. Lett. 2018, 20, 3853-3857. e) Z. Liu, X. Li, T. Zeng, K. M. Engle, ACS Catal. 2019, 9, 3260-3265. f) Z. Bai, S. Zheng, Z. Bai, F. Song, H. Wang, Q. Peng, G. Chen, G. He, ACS Catal. 2019, 9, 6502-6509. g) Y. Zhang, G. Chen, D. Zhao, Chem. Sci. 2019, 10, 7952-7957. h) J. Jeon, H. Ryu, C. Lee, D. Cho, M.-H. Baik, S. Hong J. Am. Chem. Soc. 2019, 141, 10048-10059. i) Z. Liu, J. Chen, H.-X. Lu, X. Li, Y. Gao, J. R. Coombs, M. J. Goldfogel, K. M. Engle, Angew. Chem. 2019, 131, 17224-17229; Angew. Chem. Int. Ed. 2019, 58, 17068- 17073. j) H.-Q. Ni, P. Cooper, S. Yang, F. Wang, N. Sach, P. G. Bedekar, J. S. Donaldson, M. Tran-Dubé, I. J. McAlpine, K. M. Engle, Angew. Chem. Int. Ed., 2022, e202114346.

[9] H.-Q. Ni, Z.-Q. Li, V. T. Tran, K. M. Engle, Tetrahedron 2021, 93, 132279 .

[10] a) T. Pei, R. A. Widenhoefer, J. Am. Chem. Soc. 2001, 123, 11290-11291. b) H. Qian, R. A. Widenhoefer, J. Am. Chem. Soc. 2003, 125, 2056-2057.

[11] AQ-containing alkenyl amide substrates have been previously shown to undergo $E / Z$ isomerization under $\mathrm{Pd}(\mathrm{II})$ catalysis, which explains why the d.r. values do not necessarily reflect the initial $E / Z$ ratio of the starting material. For discussion, see Refs. $8 \mathrm{e}$ and $8 \mathrm{f}$ as well as the following: R. Matsuura, M. K. Karunananda, M. Liu, N. Nguyen, D. G. Blackmond, K. M. Engle, ACS Catal. 2021, 11, 4239-4246.

[12] a) W. E. Noland, Chem. Rev. 1955, 55, 137-155. b) R. Ballini, M. Petrini, Adv. Synth. Catal. 2015, 357, 2371-2402.

[13] a) T. Deguchi, H.-L. Xin, H. Morimoto, T. Ohshima, ACS Catal. 2017, 7, 3157-3161. b) L. S. Fitzgerald, M. L. O'Duill, Chem. Eur. J. 2021, 27, 8411-8436

[14] a) I. Colomer, A. E. R. Chamberlain, M. B. Haughey, T. J. Donohoe, Nat. Rev. Chem. 2017, 1, 0088. b) T. Bhattacharya, A. Ghosh, D. Maiti, Chem. Sci. 2021, 12, 3857-3870.

[15] Analogous $\mathrm{H}$-bonding interactions from $\mathrm{Pd}(\mathrm{II})$-bound acetate have been proposed in AQ-directed anti-carbopalladation processes: J. Yang, Y. Zhang, R. Zhu, Y. Xue, J. Phys. Chem. A 2021, 125, 92679278. 


\section{Entry for the Table of Contents}

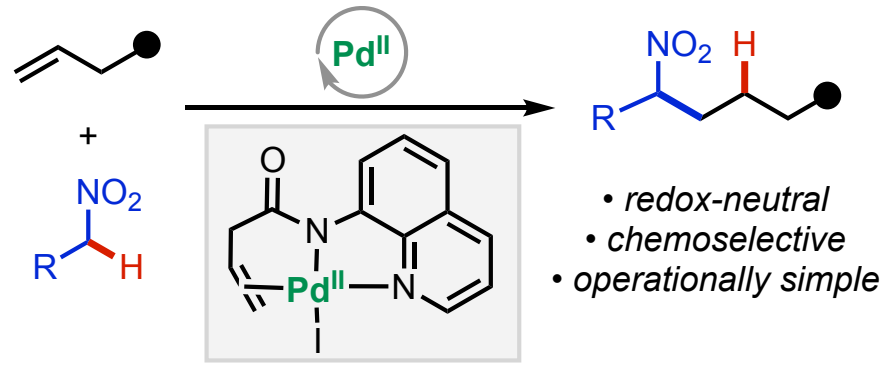

We demonstate a redox-neutral palladium-catalyzed coupling of nitroalkanes and unactivated alkenes for the synthesis of diverse range of nitro-containing products under operationally simple conditions. Synthetic transformations of the nitro group provided access to molecules that are otherwise difficult to synthesize via olefin functionalization.

Institute and/or researcher Twitter usernames: @englelab, @scrippsresearch, @ASimlandy 